\title{
Article \\ Mechanical Properties and Buckling of Kagome Graphene under Tension: A Molecular Dynamics Study
}

\author{
Trevor Wavrunek ${ }^{1}$, Qing Peng $2,3,4, * \mathbb{C}$ and Nidal Abu-Zahra ${ }^{1, * \mathbb{C}}$ \\ 1 Materials Science and Engineering Department, University of Wisconsin Milwaukee, Milwaukee, WI 53211, \\ USA; wavrune6@uwm.edu \\ 2 Physics Department, King Fahd University of Petroleum and Minerals, Dharan 31261, Saudi Arabia \\ 3 Hydrogen and Energy Storage Center, King Fahd University of Petroleum and Minerals, Dhahran 31261, \\ Saudi Arabia \\ $4 \quad$ K.A.CARE Energy Research \& Innovation Center at Dhahran, Dhahran 31261, Saudi Arabia \\ * Correspondence: qing.peng@kfupm.edu.sa (Q.P.); nidal@uwm.edu (N.A.-Z.)
}

check for updates

Citation: Wavrunek, T.; Peng, Q.; Abu-Zahra, N. Mechanical Properties and Buckling of Kagome Graphene under Tension: A Molecular Dynamics Study. Crystals 2022, 12, 292. https://doi.org/10.3390/ cryst 12020292

Academic Editor: John Parthenios

Received: 8 January 2022

Accepted: 17 February 2022

Published: 19 February 2022

Publisher's Note: MDPI stays neutral with regard to jurisdictional claims in published maps and institutional affiliations.

Copyright: (C) 2022 by the authors. Licensee MDPI, Basel, Switzerland. This article is an open access article distributed under the terms and conditions of the Creative Commons Attribution (CC BY) license (https:// creativecommons.org/licenses/by/ $4.0 /)$.

\begin{abstract}
Kagome graphene is a carbon allotrope similar to graphene, with a single-atom thickness and a co-planar atomic structure. Despite interesting electronic properties, its mechanical behavior is still elusive. We have investigated the tensile properties of Kagome graphene under various strain rates and finite temperatures using molecular dynamics simulations. The Young's modulus, ultimate tensile strength, fracture strain, and fracture toughness of the unsupported bulk material were measured as $96 \mathrm{GPa}, 43 \mathrm{GPa}, 0.05$, and $1.9 \mathrm{~J} \mathrm{~m}^{-3}$, respectively, at room temperature and a strain rate of $10^{9} \mathrm{~s}^{-1}$. Two deformation-stages were observed under tensile loading: normal and wrinkled. Initially, the Kagome graphene system stays in a co-planar structure without wrinkling until the tensile strain reaches 0.04 , where it starts to wrinkle, unlike graphene. The wrinkle wavelength and magnitude suggest a very low bending rigidity, and wrinkle formation does not follow a rate predicted by continuum mechanics. Furthermore, the fracture mechanism of wrinkled Kagome graphene is briefly discussed.
\end{abstract}

Keywords: molecular dynamics simulation; Kagome graphene; wrinkling; Griffith brittle fracture; mechanical properties

\section{Introduction}

Since its isolation and characterization in 2004 by Novosleov et al., graphene [1] has become the preeminent two-dimensional material in materials study; inspiring thousands of publications each year. Graphene is made of a single-layer honeycomb lattice of carbon atoms and has been acclaimed for its impressively large surface area $\left(2630 \mathrm{~m}^{2} / \mathrm{g}\right)$ [2], high room temperature carrier mobility $\left(200,000 \mathrm{~cm}^{2} / \mathrm{Vs}\right)$ [3], high elastic modulus (1.0 TPa) [4], high thermal conductivity $\left(5 \times 10^{3} \mathrm{~W} / \mathrm{mK}\right)$ [5] and optical transmittance ( 97.7\%) [2], making it amongst the most desirable materials for numerous applications.

The exemplary performance of graphene has inspired investigation into other materials in search of novel properties resulting from two-dimensional effects. Since the synthesis of unique two-dimensional materials is often non-trivial, advancements in computational chemistry and computational materials science have been instrumental in navigating the structure-properties-performance relationship for similar structures. These computational methods allow for the prediction of chemical, mechanical, and electrical properties of materials that are difficult or even impossible to synthesize.

One of the two-dimensional materials that has garnered recent interest, and inspired our work, is Kagome graphene (KG). KG is a two-dimensional graphene allotrope of $\mathrm{sp}^{2}$ hybridized carbon arranged in a structure of trihexagonal tiling. The structure is shown in Figure 1a and can be visualized as a series of equilateral triangles conjoined at each vertex. Kagome graphene has not yet been synthesized, but computational methods have 
predicted some of its interesting properties and its molecular-scale geometry. According to Sarikavak-Lisesivdin et al.'s ground state calculations [6], illustrated in Figure 1 and Table 1, KG is likely to have double bonds connecting the triangular cyclopropane rings, although bond order within the rings is uncertain. The bond angles are also predicted to be exactly $60^{\circ}$ for acute bonds and $150^{\circ}$ for obtuse bond angles. Despite the extreme bond angles, Chen. et al. predicted a cohesive energy of $8.26 \mathrm{eV} /$ atom and found no soft phonon modes in the Brillouin zone [7], indicating a stable structure.
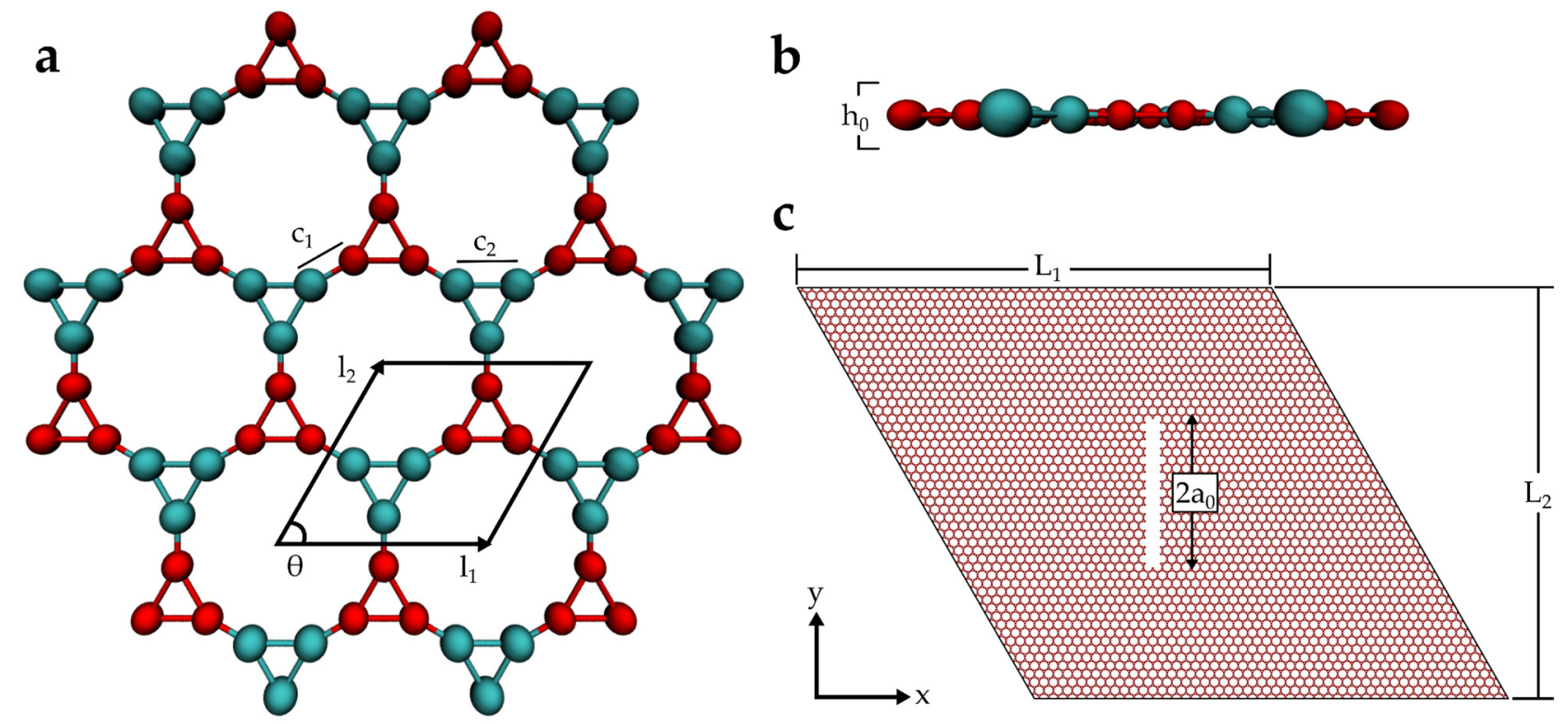

Figure 1. Kagome graphene (KG) (a) normal to two-dimensional sheet and (b) from the side. (c) Kagome graphene supercell with a crack used for tensile testing. Tensile forces were applied in the $\mathrm{x}$ (zigzag) direction.

Table 1. Bond and unit cell geometries of KG as referenced in Figure 1.

\begin{tabular}{ccc}
\hline \multirow{2}{*}{ Symbol } & $\begin{array}{c}\text { Calculated Value (This } \\
\text { Study) }\end{array}$ & Calculated Value [8] \\
\hline$\theta$ & $30.0^{\circ}$ & $30.0^{\circ}$ \\
$\mathrm{l}_{1}$ & $5.45 \AA$ & - \\
$\mathrm{l}_{2}$ & $5.45 \AA$ & - \\
$\mathrm{h}_{0}$ & $3.35 \AA$ & $3.47 \AA$ \\
\hline $\mathrm{c}_{1}$ & $1.32 \AA$ & $1.30 \AA$ \\
$\mathrm{c}_{2}$ & $1.62 \AA$ & $1.59 \AA$ \\
\hline $\mathrm{L}_{1}$ & $15.6-46.8 \mathrm{~nm}$ & $13 \mathrm{~nm}$ \\
$\mathrm{~L}_{2}$ & $13.5-40.5 \mathrm{~nm}$ & $13 \mathrm{~nm}$ \\
$\mathrm{a}_{0}$ & $1.0-6.0 \mathrm{~nm}$ & - \\
\hline
\end{tabular}

KG has attracted particular interest within the materials science community because of its predicted spin frustration and has been recognized as a system with strong correlation which is expected to exhibit a ferromagnetic flat band near the Fermi level [7]. DFT simulations of KG predict a high number of electrons within a small energy range and large "density of states". These factors might cause Wigner-crystallization, fractional quantum Hall states, or even high-temperature superconductivity [6]. Kagome lattices, in general, have been shown to exhibit exotic electronic properties due to their high frustration. This has inspired numerous studies on their superconductivity [9] and antiferromagnetism [10] as a result of their tendency to have a flat band. 
Although Kagome graphene has not been successfully synthesized yet, Chen et al. suggested a self-assembly process (from cyclopropane) on single-layer boron nitride, as the lattice mismatch is only about $2 \%$ [7]. Additionally, Pawlak et al. [11] recently published a successful synthesis process of nitrogen-doped graphene on an Ag (111) substrate, a 2D material that also has a Kagome pattern [11]. With growing interest in the synthesis of these types of material, it is evident that the actualization of materials with KG-like properties has merit, and understanding the mechanical properties of Kagome lattice structures may prove valuable.

While the electronic structure properties of KG are researched, there is scant literature on its mechanical properties and failure behavior. Molecular dynamics (MD) simulations are an attractive method to studying these properties due to their lower computational cost compared to density functional theory (DFT) methods. Because of their lower cost, simulations can be performed using a larger number of atoms, greater range of test parameters, and the implementation of lattice defects to determine the failure and fracture mechanism [12]. These features have made MD a widely used method for modeling the mechanical properties of graphene $[13,14]$. In KG, using a larger simulation size can begin to illustrate otherwise unobserved behavior under loading, such as wrinkling, which would not be observed on sub-nanometer length scales. There has been limited work on KG's mechanical properties using small (less than 1000 atom) DFT simulations [15,16]. Similarly, Zhao et al. [8] reported use of a molecular static study to test the material properties of three graphene allotropes, including "cyclicgraphene" as named, which has a similar atomic structure of KG of this study. In their study, a defect-free $13 \times 13 \mathrm{~nm}$ sheet was examined at $0 \mathrm{~K}$ with quasi-static mechanical loading. The finite temperature effect and the strain rate effect are still elusive.

In this study, we have examined the mechanical properties of Kagome graphene monolayers under finite temperatures via tensile test in molecular dynamics simulation, described in Section 2. Multiple independent variables were examined to elucidate the effects of simulation size, strain rate, and temperature. Furthermore, the simulation was utilized to illustrate wrinkling and fracture in the material, which are discussed in Section 3. The discussion and conclusions regarding the mechanical properties and fracture mechanics are finally described in Section 4.

\section{Materials and Methods}

The molecular dynamics simulations in this study are performed using the large-scale atomic/molecular massively parallel simulator (LAMMPS) [17]. Atomistic simulation results and figures were rendered using OVITO [18] and VMD [19]. The atom position and simulation cell input file, as optimized by Morresi et al. [15] through DFT calculations, was used with modifications to prevent interactions between periodic Z-axis layers. The primitive unit cell input file is included in Supplementary Materials. The inter atomic interaction is described by the AIREBO-Morse pair potential with a 3.0 A cutoff parameter as recommended by [20] for the simulation of bond breaking and formation in graphene. Non-bonding interactions like van der Waals are also described in this potential, which have been known to affect the mechanical properties of two-dimensional systems [21,22]. Besides the AIREBO-morse potential, we have examined Tersoff [23], BOP [24], REBO2 [25], and AIREBO [26] potentials. Minimization and equilibration using these potentials produced a similar atomic structure that agree well with a previous molecular dynamics study by Zhao et al. [8]. It should be considered that MD minimization results do deviate from DFT minimization as a result of high bond angle strain, which may cause variation in the calculated properties. Tensile test simulation was carried out using an isothermal-isobaric (NPT) ensemble. Tensile load was applied in the "zigzag" direction, or horizontal axis of Figure 1 [27]. A $25 \times 22.5 \times 10 \mathrm{~nm}$ parallelepiped simulation box with 15,000 carbon atoms was used as a control for simulation size, and the system was initially equilibrated at $300 \mathrm{~K}$ and $1 \mathrm{~atm}$. For tensile tests, a strain rate of $10^{9} \mathrm{~s}^{-1}$ is used when not otherwise 
noted. Periodic boundary conditions were chosen for in-plane two dimensions ( $\mathrm{x}$ and $\mathrm{y}$ directions), while the out-of-plane dimension was fixed at $5 \mathrm{~nm}$.

To study the fracture toughness of KG, a nano "crack" was generated by removing atoms near the center of the simulation area, as shown in Figure 1c. When failure occurs under a tensile load, simulation of the crack propagation in the lattice can be used to determine if a material undergoes Griffith's brittle fracture. As calculated in Zhang et al. [27], Griffith's fracture criteria are satisfied in a 2D material with a crack of length $2 a_{0}$ when the decrease in strain energy exceeds the increase in edge energy $\gamma$ of the crack. This can be written as a function of critical stress $\sigma_{\mathcal{c}}$ as shown in Equation (1). Since the terms $a_{0}$ and $\sigma_{c}$ are experimentally measurable in LAMMPS, and the right-hand side of the equation is constant for a given material, the Griffith's brittle fracture criteria can be verified for KG by measuring $\sigma_{c}$ for multiple $a_{0}$ and observing whether the critical stress intensity factor of fracture, $K_{c}=\sigma_{c} \sqrt{\pi a_{0}}$ is constant.

$$
\sigma_{c}=\sqrt{\frac{2 \gamma E}{\pi a_{0}}}
$$

\section{Results and Discussion}

\subsection{Simulation Size Effect}

Simulation size is known to affect the output of a MD simulation. Therefore, multiple simulation box sizes were first examined to determine the necessary number of atoms to emulate a bulk material. Defect-free KG sheets of size ranging from 15.6 by $13.5 \mathrm{~nm}$ to 46.8 by $40.5 \mathrm{~nm}$ (aspect ratio of 1.15:1) were observed under tensile load until fracture, as shown in Figure 2. Although the stress-strain curves of the samples did not converge, the 27 by $23.5 \mathrm{~nm}(15,000$ atoms) was the smallest simulation size to experience wrinkling at the same strain as the largest simulation size under longitudinal buckling (indicated by a dramatic decrease and subsequent increase in slope). The 25 by $22.5 \mathrm{~nm}$ simulation box started to buckle at a similar time and exhibited similar behavior until failure as the largest simulation box at a lower computation cost. A similar simulation was conducted on defect-free graphene for comparison purposes, also shown in Figure 2.

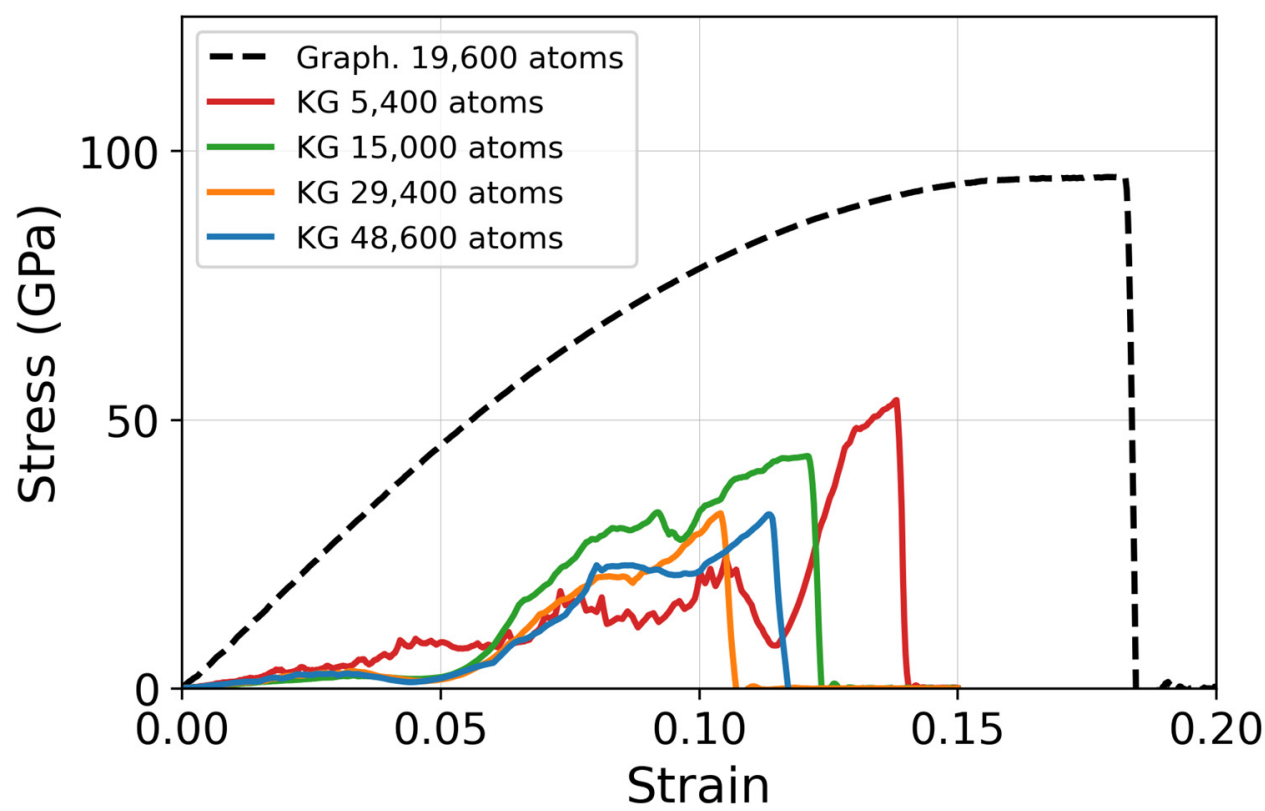

Figure 2. Stress strain relationship under tensile load of 2D single-layer Kagome graphene (KG) and traditional graphene (Graph.) sheets.

Stress-strain results show that the number of simulated atoms had a significant effect on the measured material properties of bulk KG, possibly because of wrinkling, and even- 
tually buckling of the sheet. Table 2 lists the calculated properties 40,000 atoms of graphene (Aspect ratio of 1.73:1), and 48,600 atoms of KG. These results were taken as the average from seven MD simulations using different initialization seeds. Uncertainty calculations denoting one standard deviation were included with each result. Of particular note is the large uncertainty associated with Young's modulus. This is expected, since pressure fluctuates appreciably in MD simulation, and the associated strain value is small [28]. Literature values for Zhao et al.'s calculations were also included for comparison, using the point of buckling as a failure condition for planar KG. The calculated mechanical properties show notable discrepancies for fracture stress and fracture strain. This study's calculations predict a stronger, more brittle material than Zhao et al., which is likely due to non-bonding interactions caused by the wrinkling behavior and differences in bending rigidity, which is discussed in detail in Section 3.4. Figure 3 illustrates these results and demonstrates that the 15,000 atoms simulation does not yet converge with a bulk-equivalent simulation, but it is a more appropriate approximation than the smaller simulation, especially at low strains. However, it is clear that calculated values of fracture stress and strain are most valuable to determine trends in properties under varied simulation conditions.

Table 2. Mechanical properties of planar Kagome graphene and graphene.

\begin{tabular}{cccc}
\hline Property & Graphene & KG (This Study) & KG [13] ${ }^{\mathbf{1}}$ \\
\hline Simulated atoms & 40,000 & 48,600 & 4050 \\
Young's Modulus (GPa) & $937 \pm 4$ & $96 \pm 23$ & $121.5 \pm 1.6$ \\
Ultimate Strength (GPa) & $95.2 \pm 0.1$ & $43.1 \pm 1.6$ & 8.8 \\
Strain at Failure & $0.186 \pm 0.003^{3}$ & $\sim 0.047 \pm 0.0043^{2}$ & $0.234^{3}$ \\
Toughness $\left(\mathrm{J} \mathrm{m}^{-3}\right)$ & $12.2 \pm 0.3$ & $0.124 \pm 0.0014^{3}$ & $\mathrm{NA}$ \\
\hline
\end{tabular}

1 Calculated using MD (AIREBO, 0 K, 2.0 Å cutoff). 2 Failure strain taken at approximate point of buckling. 3 Failure strain taken at point of fracture.
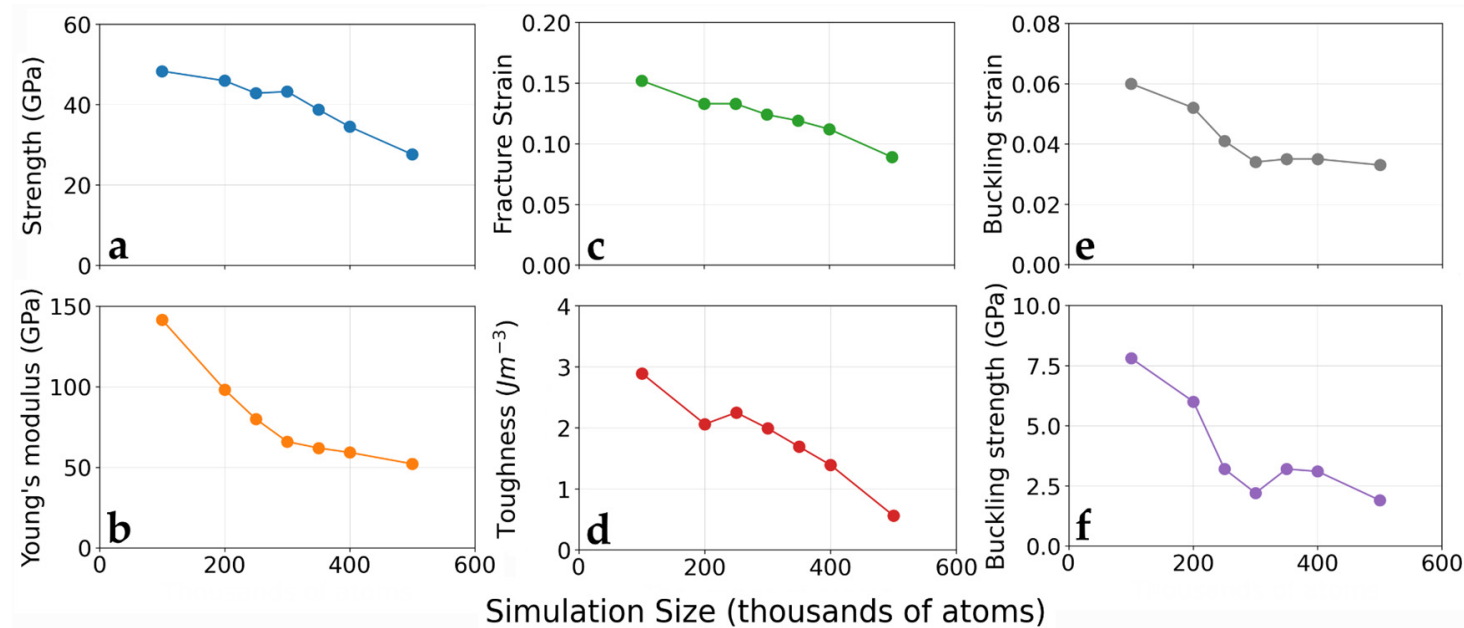

Figure 3. Mechanical properties of KG simulations with increasing number of atoms. (a) Ultimate tensile strength, (b) Young's modulus, (c) fracture strain, (d) fracture toughness, (e) strain at onset of wrinkling, and (f) stress at onset of wrinkling.

\subsection{Strain Rate Effect}

Strain rate has a considerable influence on the mechanical response of a material to loading conditions. Decreasing the strain rate is expected to decrease fracture strength and strain, and such effects are observed in graphene [29]. Strain rates between $0.0125 \%$ $\mathrm{ps}^{-1}$ and $0.20 \% \mathrm{ps}^{-1}$ were tested on KG sheets, as shown in Figures 4 and 5. From visual observations in OVITO, slower strain rates allow the KG sheet more time to buckle, and at sufficiently slow strain rates it begins to converge to an ultimate strength of approximately 
$600 \mathrm{GPa}$ and a failure strain of 0.107 . The strain rate of $010 \% \mathrm{ps}^{-1}$ provided the maximum calculated ultimate strength and maximum toughness, which are shown in Table 2.

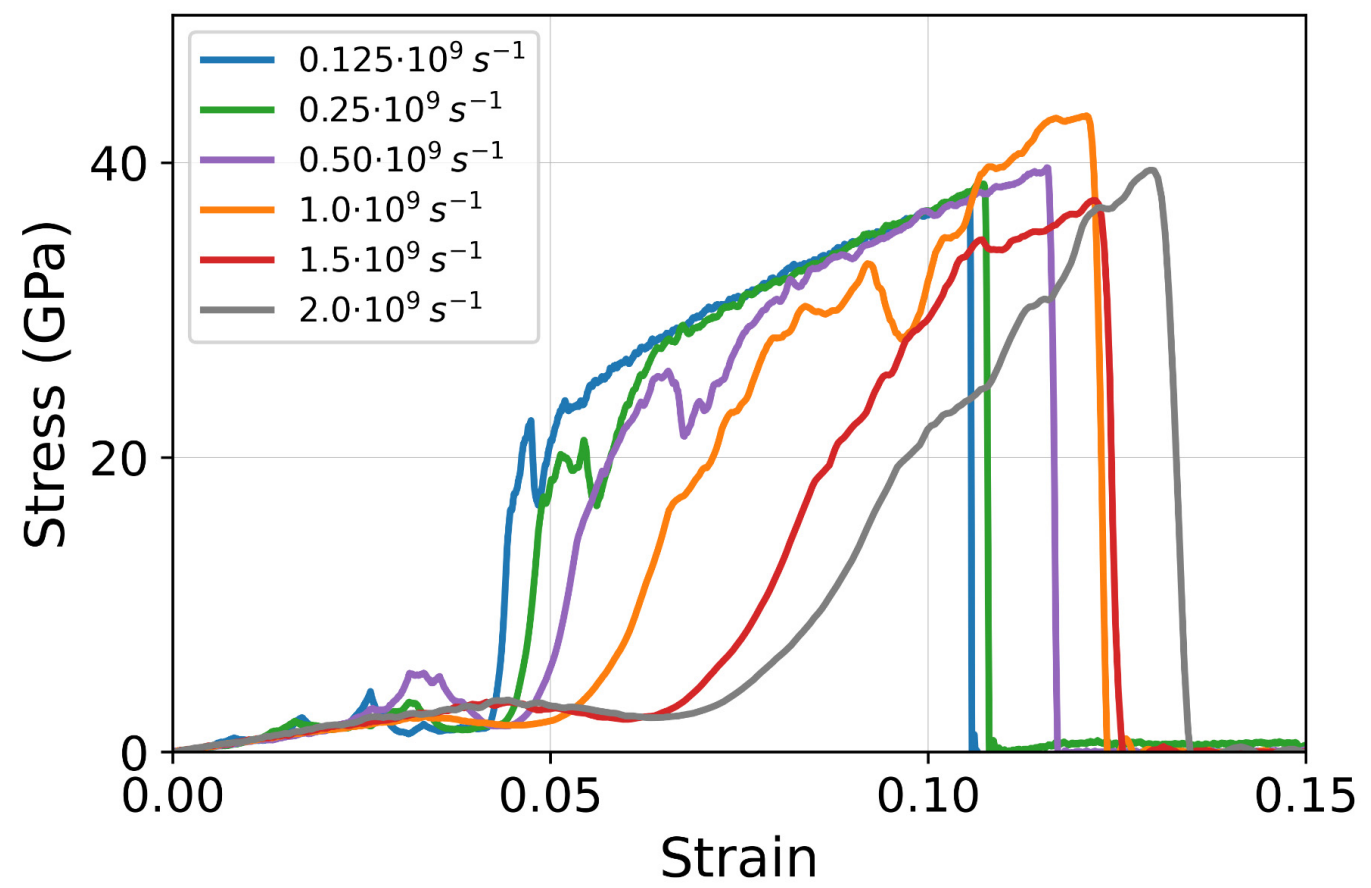

Figure 4. Strain rate effect on the stress-strain relation for KG. At low stresses, the curves begin to converge prior to fracture, suggesting an influence of buckling rate on the material properties.
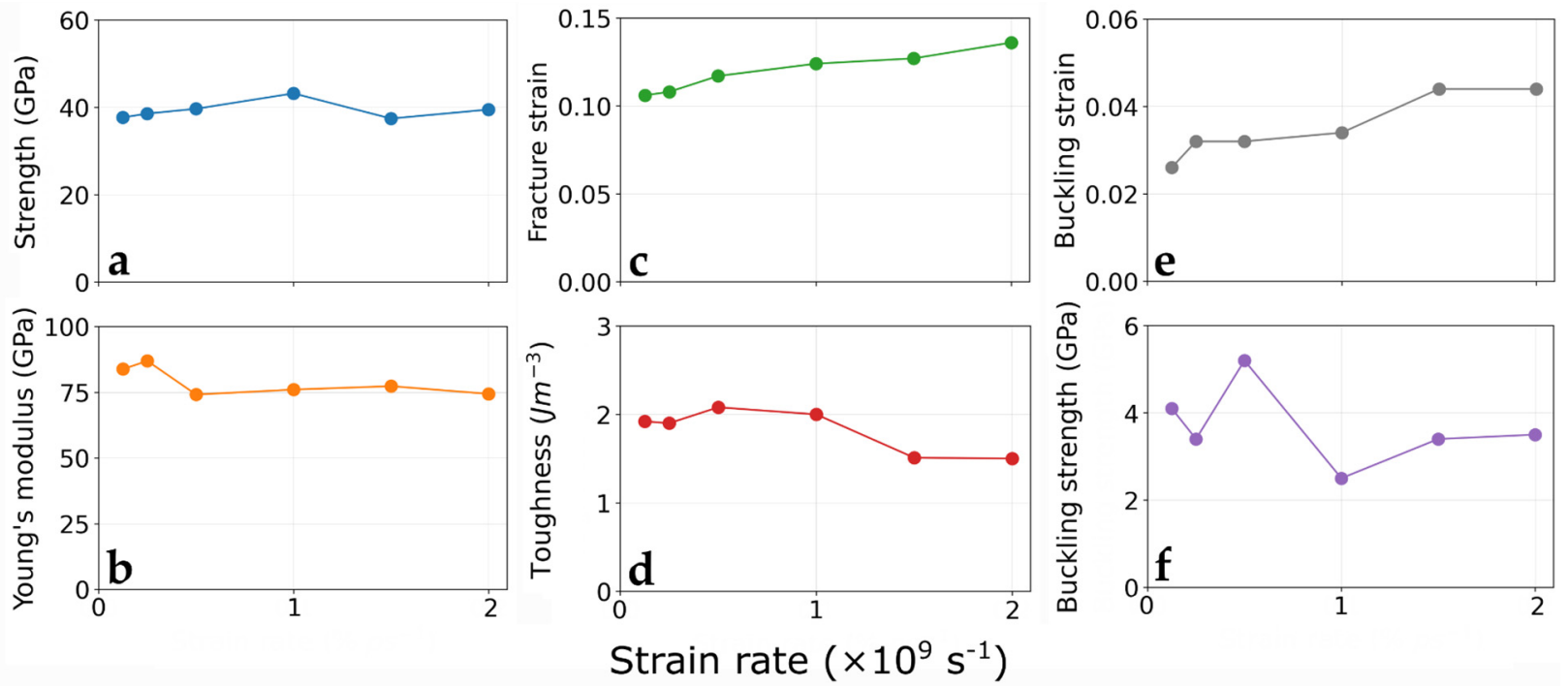

Figure 5. Mechanical properties of KG simulations with increasing strain rate. (a) Ultimate tensile strength, (b) Young's modulus, (c) fracture strain, (d) fracture toughness, (e) strain at onset of wrinkling, and (f) stress at onset of wrinkling.

As shown in Figure 5c,e, fracture and buckling strains of KG are positively correlated with the strain rate, indicating that planar KG becomes increasingly ductile under high strain rates, opposite to what would be expected for a tensile test. Likewise, the material experienced a small decrease in toughness and a negligible change in strength, which are also counter to our expectations. The unlikely outcomes of KG behavior can be explained by the onset of wrinkling in the KG sheets under high strain rates. Figure 4 shows a significant increase in stresses for the same change in strains $(0.05-0.1)$ when measured at different strain rates (low-high). 
When observed through OVITO, qualitative observations show a change in wrinkle growth rate at different strains. Buckling from tensile load is a sign of elasticity, and the sheet's high flexibility in the lateral direction seems to aid in improving buckled KG's response to high strain rates. At lower strain rates, buckling occurs at the same speed as higher strain rates, but it starts and ends at lower strains. Observing the stress-strain behavior, shown in Figure 4, under different strain rate conditions, it is evident that the buckled KG is stronger than its planar shape.

\subsection{Temperature Effect}

It is generally expected for materials to exhibit different mechanical behavior when subjected to loading conditions under higher or lower temperatures. In order to develop a more precise understanding of KG's behavior at various temperatures, the tensile test simulation was repeated at seven temperatures ranging between $100 \mathrm{~K}$ and $500 \mathrm{~K}$. As shown in Figure 6, when increasing KG's temperature, all mechanical properties decreased, indicating a more elastic, but weaker, behavior.
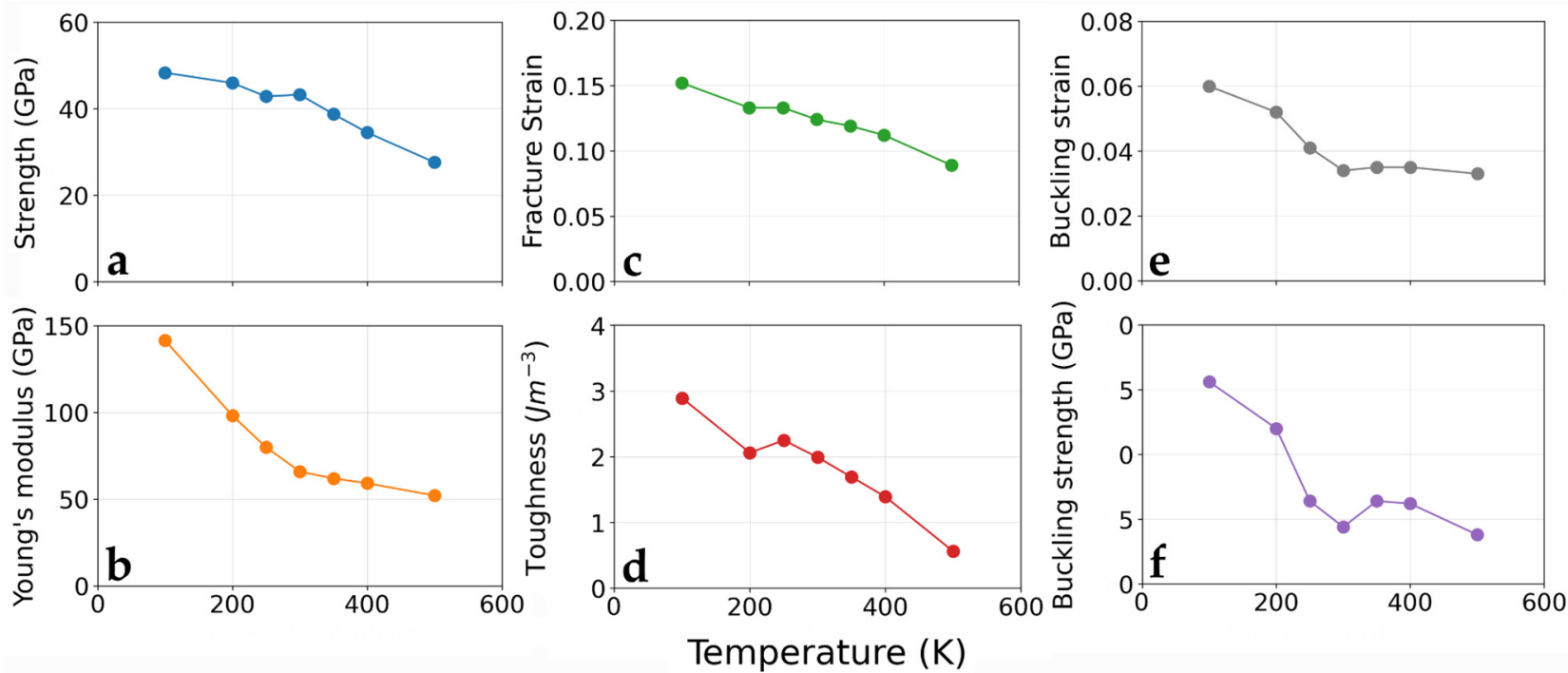

Figure 6. Mechanical properties of KG simulations with increasing temperature. (a) Ultimate tensile strength, (b) Young's modulus, (c) fracture strain, (d) fracture toughness, (e) strain at onset of wrinkling, and (f) stress at onset of wrinkling.

\subsection{Fracture Mechanism}

One of the strengths of MD is its ability to observe the failure of a material with femtosecond resolution. Since graphene is expected to undergo Griffith's fracture [20], KG was examined for this property as well. From Equation (1), KG can be said to experience Griffith's brittle fracture if its fracture toughness, $\mathrm{K}_{\mathrm{C}}$, given by $\sigma_{c} \sqrt{\pi a_{0}}$, is constant. The term $a_{0}$ refers to half of the crack length, as shown in Figure 1, and the critical stress $\left(\sigma_{c}\right)$ is measured at the initial propagation of the crack. As shown in Table 3, the fracture toughness $\left(K_{C}\right)$ changes with the initial crack length, indicating that KG does not meet the Griffith's fracture criteria [20], and instead experiences ductile failure.

Table 3. Fracture toughness of KG for an increasing crack length.

\begin{tabular}{lcccc}
\hline Crack Half-Length, $\boldsymbol{a}_{0}$ & $\mathbf{1 . 0} \mathbf{~ n m}$ & $\mathbf{2 . 0} \mathbf{~ n m}$ & $\mathbf{4 . 0} \mathbf{~ n m}$ & $\mathbf{6 . 0} \mathbf{~ n m}$ \\
\hline$K_{c}=\sigma_{c} \sqrt{\pi a_{0}}(\mathrm{MPa} \sqrt{m})$ & 1.69 & 0.952 & 0.541 & 0.373 \\
\hline
\end{tabular}

\subsection{Wrinkling}

Material wrinkling is not unique to KG and has been observed in many 2D materials, including graphene [30-37]. In practice, 2D materials often wrinkle during synthesis or 
handling, and investigation into the alternate properties of wrinkled materials is not novel. Wrinkling can be beneficial, as it is anticipated to improve a material's stability [38] and can change the band structure and electrical properties of some materials to be more desirable for applications in sensors and surface modification [39,40]. Although the important properties of KG rely on its two-dimensional band structure, electronic properties of wrinkled $\mathrm{KG}$ remain unexplored.

A comprehensive understanding of wrinkling may become valuable for KG applications. Therefore, the wrinkling-wavelength and amplitude of KG were investigated. Wrinkling in a sheet can be described in a number of ways, and is often examined first using Cerda, Ravi-Chandar, and Mahadevan's general theory of wrinkling for thin elastic materials [41,42]. This continuum mechanics-based model is the most straightforward, and has been shown to successfully predict the wrinkling behavior of multilayer graphene [31,43]. The theoretical model was developed to account for the energetic cost to create wrinkles while adhering to geometrical constraints. The derived equations for wrinkling-wavelength and -amplitude of a stretched, unsupported sheet are given by Equations (2) and (3), respectively. $L$ denotes the original sheet length, $t$ is the thickness of the sheet, $\gamma$ is the longitudinal strain, and $v$ is the Poisson's ratio of the material.

$$
\begin{gathered}
\lambda=\frac{\sqrt{2 \pi L t}}{\left[3\left(1-v^{2}\right) \gamma\right]^{1 / 4}} \\
A=\sqrt{v L t}\left[\frac{16 \gamma}{3 \pi^{2}\left(1-v^{2}\right)}\right]^{1 / 4}
\end{gathered}
$$

The resulting wavelength, $\lambda$, and amplitude, A, were calculated for the 15,000-atom simulation at control conditions as shown in Figure 7 and the changes in geometry of the simulation box was used as a foundation to calculate $L(26.4 \mathrm{~nm})$ and strain, $\gamma$. The Poisson's ratio of KG was not constant thorough the test due to buckling, and the lateral to longitudinal strain ratio increased to 10.5 when the sheet buckled. Therefore, the Poisson's ratio of the planar sheet $(v=0.440)$ as calculated by Zhao et al.'s analysis [8] was utilized. Tapaszto et al. [35] highlights the difficulty of defining $t$ for the calculation of a monolayer sheet thorough their analysis of nanoscale wrinkles in graphene. Thickness could be taken as the expected KG sheet separation thickness $\left(h_{0}=3.35 \AA\right.$ ), or as a plate with effective thickness of $0.8 \AA$ [35]. Wrinkles do not begin to form until after $4 \%$ longitudinal strain, and although the continuum model accurately predicts the initial amplitude, it does not accurately predict the amplitude nor wavelength once the wrinkles increase in scale. It is important to point out that the wrinkling could be suppressed by the small size and free-boundary conditions as implied in reference [8].
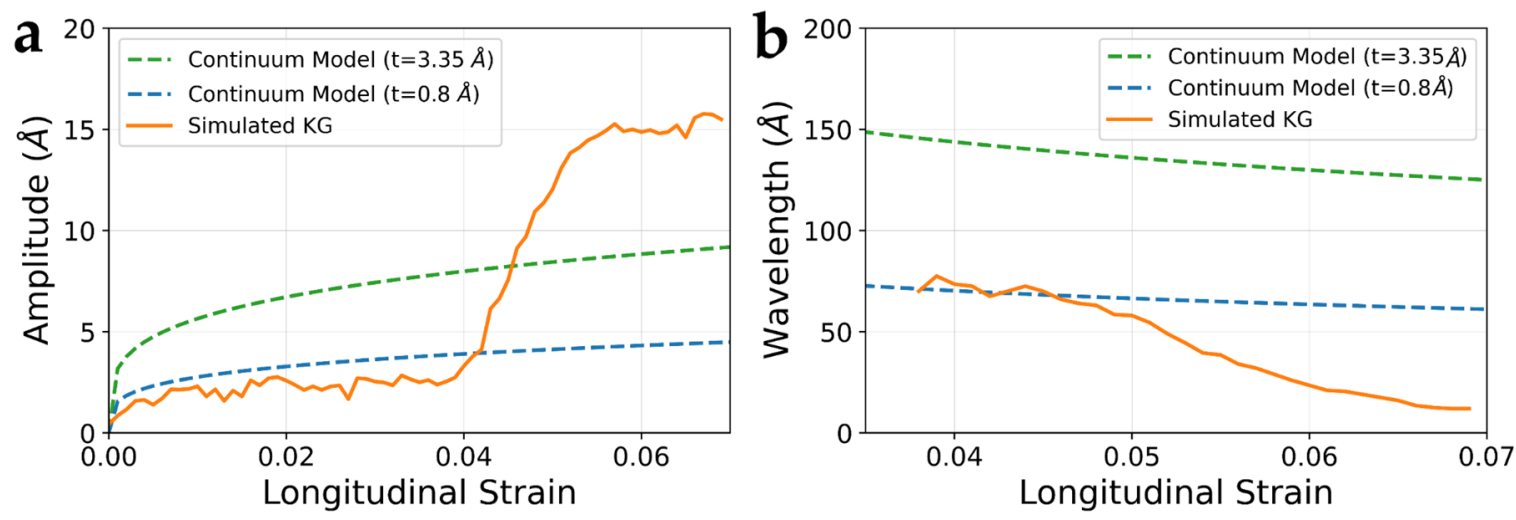

Figure 7. Predicted and observed (a) wavelength and (b) amplitude of wrinkled developed during tensile test. Corrected theoretical values were made by taking the magnitude of an imaginary output. Predicted behavior calculated from simulation box size strain and sheet thickness of $3.35 \AA$. 
It is worth noting that Equations (2) and (3) were derived using the boundary conditions of a macroscale, finite-width plate with fixed ends along the tensile axis and unsupported boundaries in the transverse direction [41]. Although these equations were designed to function away from the edges and boundaries, the periodic boundary conditions along the transverse and longitudinal directions in the simulated KG should not be ignored. The results in Figure 7 provide a similar result to [35] with graphene. KG appears to have a low bending rigidity, which significantly reduces its resistance to out-of-plane deformation. This violates the inextensible deformation assumption of [41], and may be a cause behind the unexpected behavior. Thus, Tapaszto et al.'s [35] conclusion that nanoscale wrinkling is better described via a quantum mechanical mechanism for sheets one atom in thickness instead of a continuum mechanical method is likely to apply to KG as well. When this wrinkling phenomenon occurs in unsupported $\mathrm{KG}$, the material is no longer stabilized along the plane of applied force, and buckling occurs readily along the plane as a result of increased longitudinal stress.

The evolution of wrinkles by tensile strain in similarly sized KG and graphene sheets is shown in four snapshots in Figure 8a. Graphene behaved as expected: nanoscale wrinkling as described by [35] is present, as are larger wrinkles as expected by continuum mechanics theories [34,36,39]. KG does not experience wrinkles on multiple-length scales, however, and instead spontaneously develops wrinkles much smaller than predicted by continuum theories. These qualitative results suggest an extremely low bending rigidity that allows the nanoscale wrinkles to grow in sufficient size to cause instability in the sheet, resulting in buckling, then failure.

a

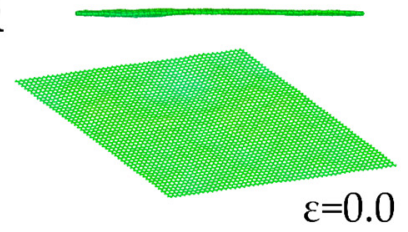

b

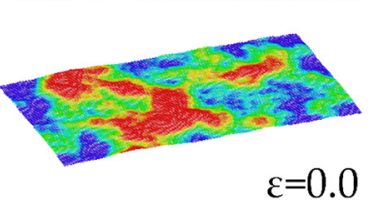

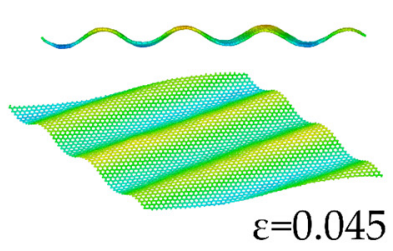

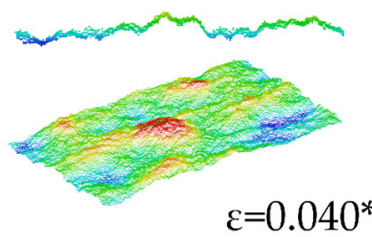

inos.
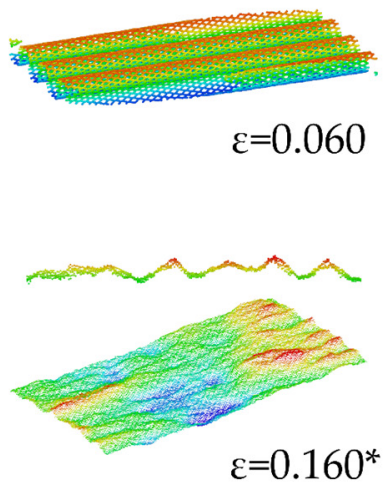
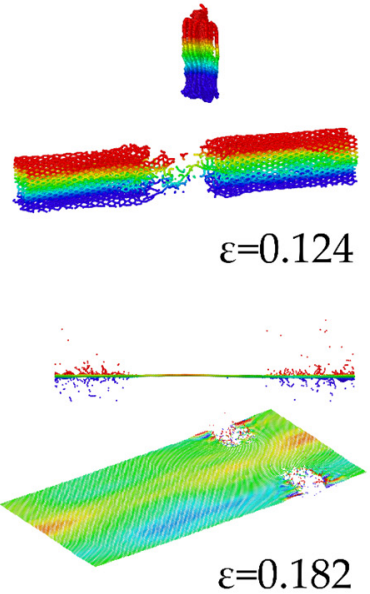

Figure 8. Evolution of wrinkles in Kagome graphene (a) compared to that of (b) graphene, with strain noted for each snapshot and cross section. A* denotes a $10 \times$ scaling of the height axis to illustrate the nanoscale wrinkles present in graphene. Initial sheet widths are $23.6 \mathrm{~nm}$ and $24.2 \mathrm{~nm}$ for $\mathrm{KG}$ and graphene respectively.

Since there are well established data for graphene's mechanical properties, we applied our simulation to graphene to validate its accuracy. Comparing the present study's calculation of mechanical properties to those of other published works showed that our simulation can accurately predict graphene's mechanical properties. For example, MD simulations of defect-free graphene at $300 \mathrm{~K}$ and $0.001 \mathrm{ps}-1$ calculated a Young's modulus of approximately $0.9 \mathrm{TPa}$ and ultimate tensile strength near $90 \mathrm{GPa}$ [29]. The present study simulated graphene with a modulus of $0.94 \mathrm{TPa}$ and strength of $95.2 \mathrm{GPa}$.

While this study predicted properties within acceptable levels of error for graphene, KG behaved fundamentally differently than when observed in smaller simulations. Although Young's modulus values calculated in this study are corroborated by Zhao et al.'s simulation, the extreme and spontaneous wrinkling observed in this unsupported system caused significantly different results. Similar experiments have been conducted on folded 
graphene, or "grafolds" [44]. In this alternative structure, graphene was observed to be more rigid, which is in part corroborated by studies showing that bilayer graphene is less elastic than monolayer graphene [27]. However, grafold structures were not observed to possess the greater tensile strength seen in wrinkled KG. Graphene's lower bending rigidity causes grafold structures to fracture at the junction between curved and planar sections of the sheet, while KG instead fractures at the center of the sheet. This distinction may contribute to the unexpected increase in fracture strength observed in KG.

It should be noted that in comparison to graphene, which has numerous routes to synthesis, KG, has not been synthesized yet and cannot be tested experimentally. To the best of the authors' knowledge, no previous study has examined KG at energies above its ground state configuration, and a further understanding of this material at finite temperatures would be valuable. Considering the results of this study, the development of a pair potential in DFT specifically for KG would be necessary to verify these results.

\section{Conclusions}

We have investigated the mechanical properties of KG at finite temperatures and various strain rates using molecular dynamics simulation. With an applied periodic boundary condition, we have observed that KG begins to wrinkle, and eventually buckle, under tensile stresses before exhibiting ductile failure. The bucking improves the fracture toughness and strength. The fracture strain and fracture toughness are especially sensitive to the strain rates, and material properties react linearly to changes in temperature between $100 \mathrm{~K}$ and $500 \mathrm{~K}$ by becoming weaker and more ductile. KG behaved unexpectedly in response to a tensile load, and did not follow the behavior predicted by a continuum mechanics-based wrinkling model. Furthermore, KG did not experience the same type of stable nanoscale wrinkles as seen in graphene, and instead became unstable once wrinkling was onset. Additionally, by testing the material for Griffith's brittle fracture, KG was compared to graphene beyond mechanical properties. Although it shares a similar structure with graphene, KG appears to experience ductile failure instead of brittle fracture.

Although MD methods were unable to emulate ab initio DFT methods, conclusions can still be drawn within the trends observed. This work demonstrates that bulk KG can be simulated at finite temperatures using MD with a reasonable degree of accuracy, which may be useful in future examinations of large-scale defects or in applications of the material. The impressive properties predicted for buck KG and observed deviations from ab initio predictions warrant further MD simulations to investigate its properties after development of a dedicated pair potential. The results of these experiments should inform interest in further research of this potentially valuable two-dimensional material.

Supplementary Materials: The following are available online at https:/ /www.mdpi.com/article/10 .3390 / cryst12020292/s1, LAMMPS input file of KG unit cell, Video S1: Render of buckling in KG during tensile test

Author Contributions: Conceptualization, Q.P.; methodology, Q.P. and T.W.; software, Q.P. and T.W.; formal analysis, T.W.; investigation, T.W.; resources, Q.P. and N.A.-Z.; writing-original draft preparation, T.W.; writing-review and editing, T.W., Q.P. and N.A.-Z.; visualization, T.W.; supervision, Q.P. and N.A.-Z.; project administration, Q.P.; funding acquisition, N.A.-Z. All authors have read and agreed to the published version of the manuscript.

Funding: Funding for the simulation work was provided by the Materials Science and Engineering Department at the University of Wisconsin-Milwaukee (UWM). Q.P. would like to acknowledge the support provided by the Deanship of Scientific Research (DSR) at King Fahd University of Petroleum and Minerals (KFUPM) through the K.A.CARE project (KACARE211-RFP-02).

Data Availability Statement: The data presented in this study are available on request.

Conflicts of Interest: The authors declare no conflict of interest. The funders had no role in the design of the study; in the collection, analyses, or interpretation of data; in the writing of the manuscript, or in the decision to publish the results. 


\section{References}

1. Novoselov, K.S.; Geim, A.K.; Morozov, S.V.; Jiang, D.; Zhang, Y.; Dubonos, S.V.; Grigorieva, I.V.; Firsov, A.A. Electric Field Effect in Atomically Thin Carbon Films. Science 2004, 306, 666-669. [CrossRef] [PubMed]

2. Zhu, Y.; Murali, S.; Cai, W.; Li, X.; Suk, J.W.; Potts, J.R.; Ruoff, R.S. Graphene and Graphene Oxide: Synthesis, Properties, and Applications. Adv. Mater. 2010, 22, 3906-3924. [CrossRef] [PubMed]

3. Morozov, S.V.; Novoselov, K.S.; Katsnelson, M.I.; Schedin, F.; Elias, D.C.; Jaszczak, J.A.; Geim, A.K. Giant Intrinsic Carrier Mobilities in Graphene and Its Bilayer. Phys. Rev. Lett. 2008, 100, 016602. [CrossRef] [PubMed]

4. Lee, C.; Wei, X.; Kysar, J.W.; Hone, J. Measurement of the Elastic Properties and Intrinsic Strength of Monolayer Graphene. Science 2008, 321, 385-388. [CrossRef]

5. Balandin, A.A.; Ghosh, S.; Bao, W.; Calizo, I.; Teweldebrhan, D.; Miao, F.; Lau, C.N. Superior Thermal Conductivity of Single-Layer Graphene. Nano Lett. 2008, 8, 902-907. [CrossRef]

6. Sarikavak-Lisesivdin, B.; Lisesivdin, S.B.; Ozbay, E.; Jelezko, F. Structural Parameters and Electronic Properties of 2D Carbon Allotrope: Graphene with a Kagome Lattice Structure. Chem. Phys. Lett. 2020, 760, 138006. [CrossRef]

7. Chen, Y.; Sun, Y.Y.; Wang, H.; West, D.; Xie, Y.; Zhong, J.; Meunier, V.; Cohen, M.L.; Zhang, S.B. Carbon Kagome Lattice and Orbital-Frustration-Induced Metal-Insulator Transition for Optoelectronics. Phys. Rev. Lett. 2014, 113, 085501. [CrossRef]

8. Zhao, J.; Wei, N.; Fan, Z.; Jiang, J.-W.; Rabczuk, T. The Mechanical Properties of Three Types of Carbon Allotropes. Nanotechnology 2013, 24, 095702. [CrossRef]

9. Jiang, Y.-X.; Yin, J.-X.; Denner, M.M.; Shumiya, N.; Ortiz, B.R.; Xu, G.; Guguchia, Z.; He, J.; Hossain, M.S.; Liu, X.; et al. Unconventional Chiral Charge Order in Kagome Superconductor KV3Sb5. Nat. Mater. 2021, 20, 1353-1357. [CrossRef]

10. Jiang, N.; Ramanathan, A.; Bacsa, J.; La Pierre, H.S. Synthesis of a D1-Titanium Fluoride Kagome Lattice Antiferromagnet. Nat. Chem. 2020, 12, 691-696. [CrossRef]

11. Pawlak, R.; Liu, X.; Ninova, S.; D’Astolfo, P.; Drechsel, C.; Liu, J.-C.; Häner, R.; Decurtins, S.; Aschauer, U.; Liu, S.-X.; et al. On-Surface Synthesis of Nitrogen-Doped Kagome Graphene. Angew. Chem. Int. Ed. 2021, 60, 8370-8375. [CrossRef] [PubMed]

12. Lei, S.; Cao, Q.; Geng, X.; Yang, Y.; Liu, S.; Peng, Q. The Mechanical Properties of Defective Graphyne. Crystals 2018, 8, 465. [CrossRef]

13. Shi, T.; Peng, Q.; Bai, Z.; Gao, F.; Igor, J. Proton Irradiation of Graphene: Insights from Atomistic Modeling. Nanoscale 2019, 11, 20754-20765. [CrossRef] [PubMed]

14. Hou, J.; Deng, B.; Zhu, H.; Lan, Y.; Shi, Y.; De, S.; Liu, L.; Chakraborty, P.; Gao, F.; Peng, Q. Magic Auxeticity Angle of Graphene. Carbon 2019, 149, 350-354. [CrossRef]

15. Morresi, T.; Pedrielli, A.; a Beccara, S.; Gabbrielli, R.; Pugno, N.M.; Taioli, S. Structural, Electronic and Mechanical Properties of All-Sp2 Carbon Allotropes with Density Lower than Graphene. Carbon 2020, 159, 512-526. [CrossRef]

16. Sun, H.; Mukherjee, S.; Daly, M.; Krishnan, A.; Karigerasi, M.H.; Singh, C.V. New Insights into the Structure-Nonlinear Mechanical Property Relations for Graphene Allotropes. Carbon 2016, 110, 443-457. [CrossRef]

17. Plimpton, S. Fast Parallel Algorithms for Short-Range Molecular Dynamics. J. Comput. Phys. 1995, 117, 1-19. [CrossRef]

18. Stukowski, A. Visualization and Analysis of Atomistic Simulation Data with OVITO-the Open Visualization Tool. Model. Simul. Mater. Sci. Eng. 2010, 18, 015012. [CrossRef]

19. Humphrey, W.; Dalke, A.; Schulten, K. VMD: Visual Molecular Dynamics. J. Mol. Graph. 1996, 14, 33. [CrossRef]

20. O'Connor, T.C.; Andzelm, J.; Robbins, M.O. AIREBO-M: A Reactive Model for Hydrocarbons at Extreme Pressures. J. Chem. Phys. 2015, 142, 024903. [CrossRef]

21. Peng, Q.; Wang, G.; Liu, G.-R.; De, S. Van Der Waals Density Functional Theory VdW-DFq for Semihard Materials. Crystals 2019, 9, 243. [CrossRef]

22. Peng, Q.; Chen, Z.; De, S. A Density Functional Theory Study of the Mechanical Properties of Graphane with van Der Waals Corrections. Mech. Adv. Mater. Struct. 2015, 22, 717-721. [CrossRef]

23. Kınacı, A.; Haskins, J.B.; Sevik, C.; Çă̆ın, T. Thermal Conductivity of BN-C Nanostructures. Phys. Rev. B 2012, 86, 115410. [CrossRef]

24. Zhou, X.W.; Ward, D.K.; Foster, M.E. An Analytical Bond-Order Potential for Carbon. J. Comp. Chem. 2015, 36, 1719-1735. [CrossRef]

25. Brenner, D.W.; Shenderova, O.A.; Harrison, J.A.; Stuart, S.J.; Ni, B.; Sinnott, S.B. A Second-Generation Reactive Empirical Bond Order (REBO) Potential Energy Expression for Hydrocarbons. J. Phys. Condens. Matter 2002, 14, 783-802. [CrossRef]

26. Stuart, S.J.; Tutein, A.B.; Harrison, J.A. A Reactive Potential for Hydrocarbons with Intermolecular Interactions. J. Chem. Phys. 2000, 112, 6472-6486. [CrossRef]

27. Zhang, P.; Ma, L.; Fan, F.; Zeng, Z.; Peng, C.; Loya, P.E.; Liu, Z.; Gong, Y.; Zhang, J.; Zhang, X.; et al. Fracture Toughness of Graphene. Nat. Commun. 2014, 5, 3782. [CrossRef]

28. Wan, S.; Sinclair, R.C.; Coveney, P.V. Uncertainty Quantification in Classical Molecular Dynamics. Philos. Trans. R. Soc. A Math. Phys. Eng. Sci. 2021, 379, 20200082. [CrossRef]

29. Zhao, H.; Aluru, N.R. Temperature and Strain-Rate Dependent Fracture Strength of Graphene. J. Appl. Phys. 2010, 108, 064321. [CrossRef]

30. Wang, C.; Lan, L.; Tan, H. The Physics of Wrinkling in Graphene Membranes under Local Tension. Phys. Chem. Chem. Phys. 2013, 15, 2764-2773. [CrossRef] 
31. Zhao, C.; Liu, F.; Kong, Z.; Yan, T.; Ding, F. The Wrinkle Formation in Graphene on Transition Metal Substrate: A Molecular Dynamics Study. Int. J. Smart Nano Mater. 2021, 3, 277-287. [CrossRef]

32. Lui, C.H.; Liu, L.; Mak, K.F.; Flynn, G.W.; Heinz, T.F. Ultraflat Graphene. Nature 2009, 462, 339-341. [CrossRef] [PubMed]

33. Rakshit, B.; Mahadevan, P. Absence of Rippling in Graphene under Biaxial Tensile Strain. Phys. Rev. B 2010, 82, 153407. [CrossRef]

34. Bao, W.; Miao, F.; Chen, Z.; Zhang, H.; Jang, W.; Dames, C.; Lau, C.N. Controlled Ripple Texturing of Suspended Graphene and Ultrathin Graphite Membranes. Nat. Nanotechnol. 2009, 4, 562-566. [CrossRef]

35. Tapasztó, L.; Dumitrică, T.; Kim, S.J.; Nemes-Incze, P.; Hwang, C.; Biró, L.P. Breakdown of Continuum Mechanics for NanometreWavelength Rippling of Graphene. Nat. Phys. 2012, 8, 739-742. [CrossRef]

36. Wei, Y.; Wang, B.; Wu, J.; Yang, R.; Dunn, M.L. Bending Rigidity and Gaussian Bending Stiffness of Single-Layered Graphene. Nano Lett. 2013, 13, 26-30. [CrossRef]

37. Shoaib, H.; Peng, Q.; Alsayoud, A.Q. Atomic Insights into Fracture Characteristics of Twisted Tri-Layer Graphene. Crystals 2021, 11, 1202. [CrossRef]

38. Fasolino, A.; Los, J.H.; Katsnelson, M.I. Intrinsic Ripples in Graphene. Nat. Mater. 2007, 6, 858-861. [CrossRef]

39. Chen, Y.; Xu, S.; Xie, Y.; Zhong, C.; Wu, C.; Zhang, S.B. Ferromagnetism and Wigner Crystallization in Kagome Graphene and Related Structures. Phys. Rev. B 2018, 98, 035135. [CrossRef]

40. Duan, W.H.; Gong, K.; Wang, Q. Controlling the Formation of Wrinkles in a Single Layer Graphene Sheet Subjected to In-Plane Shear. Carbon 2011, 49, 3107-3112. [CrossRef]

41. Cerda, E.; Mahadevan, L. Geometry and Physics of Wrinkling. Phys. Rev. Lett. 2003, 90, 074302. [CrossRef] [PubMed]

42. Cerda, E.; Ravi-Chandar, K.; Mahadevan, L. Wrinkling of an Elastic Sheet under Tension. Nature 2002, 419, 579-580. [CrossRef] [PubMed]

43. Deng, S.; Berry, V. Wrinkled, Rippled and Crumpled Graphene: An Overview of Formation Mechanism, Electronic Properties, and Applications. Mater. Today 2016, 19, 197-212. [CrossRef]

44. Grafold-Zheng, Y.; Wei, N.; Fan, Z.; Xu, L.; Huang, Z. Mechanical Properties of Grafold: A Demonstration of Strengthened Graphene. Nanotechnology 2011, 22, 479501. [CrossRef] 